\title{
Dihydropyrimidine dehydrogenase polymorphisms and fluoropyrimidine toxicity: ready for routine clinical application within personalized medicine?
}

\author{
Marzia Del Re • Antonello Di Paolo • \\ Ron H. van Schaik • Guido Bocci • Paolo Simi • \\ Alfredo Falcone • Romano Danesi \\ Received: 4 May 2010 / Accepted: 29 June 2010/Published online: 25 July 2010 \\ (C) European Association for Predictive, Preventive and Personalised Medicine 2010
}

\begin{abstract}
Fluoropyrimidines, including 5-fluorouracil (5FU), are widely used in the treatment of solid tumors and remain the backbone of many combination regimens. Despite their clinical benefit, fluoropyrimidines are associated with gastrointestinal and hematologic toxicities, which often lead to treatment discontinuation. 5-FU undergoes complex metabolism, dihydropyrimidine dehydrogenase (DPD) being the rate-limiting enzyme of inactivation of 5-FU and its prodrugs. Several studies have demonstrated significant associations between severe toxicities by fluoropyrimidines and germline polymorphisms of DPD gene. To date, more than 30 SNPs and deletions have been identified within DPD, the majority of these variants having no functional
\end{abstract}

R. Danesi is Italian national representative of EPMA

M. Del Re · A. Di Paolo $\cdot$ G. Bocci $\cdot$ R. Danesi $(\bowtie)$

Division of Pharmacology, Department of Internal Medicine,

University of Pisa,

55, Via Roma,

56126 Pisa, Italy

e-mail: danesi@epmanet.eu

R. H. van Schaik

Department of Clinical Chemistry,

Erasmus University Medical Center,

Rotterdam, The Netherlands

P. Simi

Unit of Cytogenetics and Molecular Genetics,

University Hospital,

Pisa, Italy

\section{A. Falcone}

Division of Oncology, Department of Oncology,

Transplants and Advanced Technologies in Medicine,

University of Pisa,

Pisa, Italy consequences on enzymatic activity. However, the identification of deficient DPD genotypes may help identify poor-metabolizer patients at risk of developing potentially life-threatening toxicities after standard doses of fluoropyrimidines.

Keywords Fluoropyrimidines · Toxicity · DPD ·

Polymorphisms $\cdot$ Personalized medicine

\section{Introduction}

The use of fluoropyrimidines in the clinical setting

Fluoropyrimidines, including 5-fluorouracil (5-FU) and its prodrug capecitabine, are widely used drugs to treat cancer of the head and neck, cervix, breast and gastrointestinal tract, alone or in combination with other pharmacological agents, including irinotecan, oxaliplatin, cyclophosphamide, epirubicin and the monoclonal antibodies cetuximab and bevacizumab. Owing to the widespread use of these drugs, severe toxicities are induced by fluoropyrimidines, including nausea/vomiting, diarrhea, mucositis, alopecia, myelosuppression, cardiac toxicity and hand-foot syndrome (HFS) [1, 2]. These toxicities may limit treatment effectiveness due to delay in drug administration and/or treatment discontinuation; therefore the therapeutic benefit of fluoropyrimidines is compromised. Capecitabine seems to display a different profile of toxicity compared to 5-FU, being characterized by a better tolerability, but higher incidence of HFS [3]. Finally, the oral administration has some advantage, particularly in the quality of life of patients, and for this reason its use is becoming more diffuse in USA and Europe. 
Mechanism of action and metabolism of fluoropyrimidines

The pharmacological activity of fluoropyrimidines (Fig. 1) depends on the transformation of 5-FU into his active metabolite 5-fluoro-deoxyuridine monophosphate (5FdUMP), which forms a stable complex with reduced folates and thymidylate synthase (TS), the key enzyme for de novo synthesis of thymidine. The inhibition of TS leads to the arrest of DNA synthesis, an effect further enhanced by the intracellular generation of fluoropyrimidine deoxynucleotide analogues which are incorporated into DNA and RNA leading to their disruption $[4,5]$. It is worth noting that the activity of fluoropyrimidines depends on the equilibrium between anabolic and catabolic pathways. In particular, more than $80 \%$ of an administered dose of 5-FU is transformed to inactive metabolites, being the remaining $20 \%$ or less responsible for the therapeutic effect (Fig. 1). The first step of 5-FU biotransformation is catalyzed by the enzyme dihydropyrimidine dehydrogenase (DPD, Fig. 2), which is also involved in the catabolism of thymine and uracil. The degradation of uracil is responsible for the endogenous biosynthesis of $\beta$-alanine, a structural analogue of two inhibitory neurotransmitters, glycine and $\gamma$ aminobutyric acid (GABA). 5-FU is transformed by DPD into 5-fluoro-5,6-dihydrouracil (5-FDHU), which is further metabolized to $\alpha$-fluoro- $\beta$-alanine (FBAL) by two additional enzymes and finally excreted by the kidneys. The profound deficit in DPD activity is a well known metabolic syndrome of children characterized by thymineuraciluria, mental retardation and high levels of thymine and uracil in blood, urines and cerebrospinal fluid. Therefore, the administration of fluoropyrimidines to patients with a deficient DPD activity potentially results

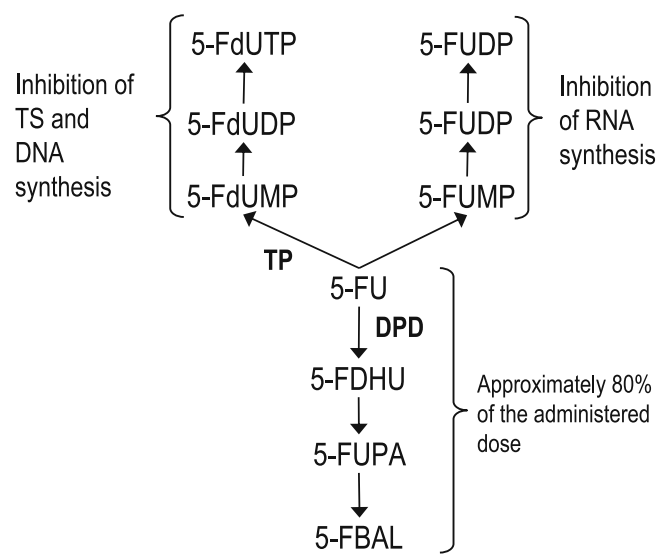

Fig. 1 Metabolic pathway of of 5-FU. 5-FdUMP/UDP/UTP: 5fluorodeoxyuridine-mono/di/triphosphate; 5-FUMP/UDP/UTP: 5fluorouridine-mono/di/triphosphate; 5- $\mathrm{FUH}_{2}$ : 5,6-dihydro-5-fluorouracil FUPA: $\alpha$-fluoro- $\beta$-ureidopropionic acid; FBAL: $\alpha$-fluoro- $\beta$-alanine; DPD: dihydropyrimidine dehydrogenase; TP: thymidine phosphorylase; TS: thymidylate synthase

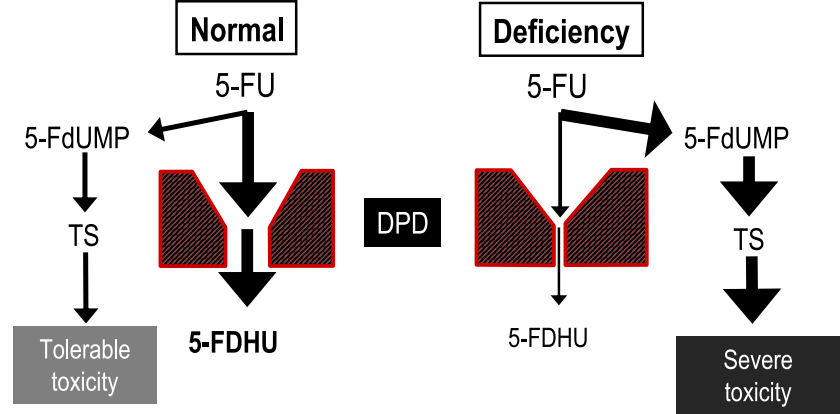

Fig. 2 DPD-dependent inactivation of 5-FU and effects of DPD deficiency. For abbreviations, see Fig. 1

in life-threatening toxicities, because a larger amount of 5FU is activated to cytotoxic metabolites (Fig. 2). Capecitabine is an orally administered inactive prodrug that is enzymatically converted into 5-FU [6]. Thymidine phosphorylase (TP) is expressed at high levels in the liver and many tumors and catalyzes the bioactivation of capecitabine preferentially in cancer tissue, leading to high concentrations of 5-FU in tumor cells [3,5].

The clinical relevance of fluoropyrimidine toxicity

Several case reports described severe toxicities in patients after the administration of standard doses 5-FU [7-9]. It is likely that a significant proportion of these adverse drug reactions are due to genetically-based differences between individuals in the response to 5-FU. Therefore, the prevention of life-threatening 5-FU toxicities, particularly in the adjuvant setting, is of fundamental clinical value. For the past 20 years, the association between DPD deficiency and severe toxicity after 5 -FU treatment, including grade 4 toxicity and death, has been extensively studied. The frequency of low DPD enzymatic activity in the general population was initially estimated between $3 \%$ and $5 \%$ $[10,11]$, although a significant ethnic variability was observed. Indeed, phenotypic and genotypic analyses of Asian [12-15], African [16], European Caucasian [17] and American [18] populations found varying frequencies of DPD deficiency. In particular, African-Americans display significantly lower DPD activity and higher prevalence of DPD deficiency than Caucasians [19], whereas higher value for DPD activity was found in a cohort of Korean subjects. A critical issue is represented by the lack of a current consensus definition of deficiency; some have proposed the use of the lower 95th percentile as threshold, while others suggested the lower 70th percentile of DPD activity from a normal population [20]. Whatever the value used to identify a DPD deficient patient could be, it is worth noting that most patients have no symptoms of partial DPD deficiency until they are challenged with a fluoropyrimidine. 


\section{Clinical features of DPD-dependent fluoropyrimidine toxicity}

The typical toxicity in a poor or null DPD metabolizer occurs at the first cycle of chemotherapy and it is characterized by grade 4 (WHO) symptoms and potentially death. Most frequent reported side effects are diarrhea grade 3 or 4 , complete alopecia, mucositis grade 3 or 4, hand-foot syndrome (Fig. 3) and neutropenia grade 3-4 [21]. Shahrokni et al. reported a case of a patient who developed recurrent chest pain and ischemic electrocardiogram changes after treatment with 5-FU and capecitabine. Cardiotoxicity associated with 5-FU and capecitabine administration is infrequently reported in the literature and appears to be dose and schedule-dependent [22]. Öfverholm et al. described a patient given adjuvant chemotherapy of weekly bolus injections of $500 \mathrm{mg} / \mathrm{m}^{2} 5-\mathrm{FU}$ and $20 \mathrm{mg} / \mathrm{m}^{2}$ folinic acid; after being asymptomatic throughout the first 2 cycles, on days 3-5 after the third cycle he experienced mild stomatitis and watering eyes. The symptoms rapidly worsened and he was admitted to hospital care for 3 weeks with grade 3-4 oral mucositis and grade 3-4 diarrhea. After 1 month of recovery, the treatment was reintroduced with a $50 \%$ dose reduction, a dose he could tolerate with only minor degree of toxicity until the end of the treatment period [23]. Another patient treated with adjuvant 5-FU $500 \mathrm{mg} / \mathrm{m}^{2}$ i.v. and folinic acid $60 \mathrm{mg} / \mathrm{m}^{2}$ i.v. on days 1 and 2 , every 2 weeks, experienced grade 3 severe fatigue and grade 2 watering eyes after two thirds of treatment [23]. Morel et al. described a patient who died from severe, polyvisceral failure following the first administration of 5FU $400 \mathrm{mg} / \mathrm{m}^{2}$ i.v. bolus, and $22 \mathrm{~h}$-infusion of 5 -FU $600 \mathrm{mg} / \mathrm{m}^{2}$, plus $400 \mathrm{mg} / \mathrm{m}^{2}$ leucovorin. Seven days after the first cycle, he was hospitalized because of grade 4 diarrhea, mucositis, grade 4 leuko/thrombocytopenia, and

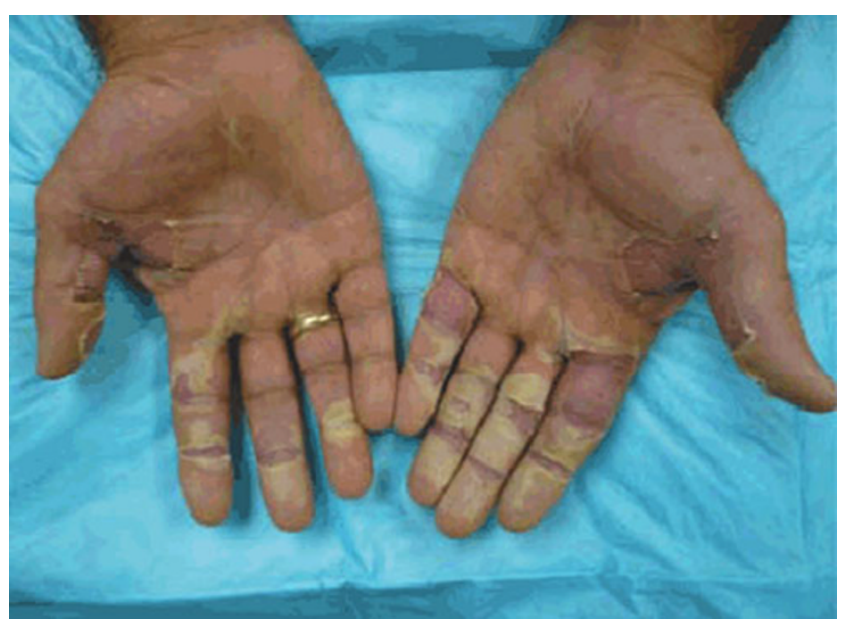

Fig. 3 Macroscopic appearance of a palmar region of a patient with severe hand foot syndrome dehydration. He then developed renal insufficiency and died 7 days later, 19 days after 5-FU administration, despite intensive supportive treatment [24].

\section{Methods for the assessment of DPD deficiency}

In vitro enzymatic activity of DPD

The first attempt to investigate DPD deficiency consisted of the assay of DPD activity in tissues and cells or by measuring levels of by-products of the enzymatic reaction. It has been demonstrated that liver DPD activity is associated with 5-FU tolerability and it is related with enzyme activity measured in mononuclear cells of peripheral blood (PBMC) [25, 26], which have been used since then as a surrogate for total body DPD. However, the application of this method into clinical practice is labor intensive since PBMCs are incubated ex vivo with radiolabelled 5-FU and the resulting rate of catabolite formation is measured by high-performance liquid chromatography (HPLC) [27, 28]. It is likely that the need for specialized technical skills and appropriate equipment have limited the clinical availability of the phenotypic assay. In addition to this, sample handling and processing are critical issues and DPD levels in PBMCs may be even influenced by the cellular heterogeneity of the sample since enzyme content may vary among monocytes, lymphocytes, and neutrophils [29]. Since the amount of DPD in tissues is dependent on the transcriptional activity of the gene, real-time quantitative PCR analysis of DPD mRNA has been proposed as a surrogate marker of DPD activity. However, the correlation between cellular DPD mRNA expression and DPD enzyme activity was disappointing since conflicting data were published in the literature [30-33]. Indeed, DPD deficiency in patients could be caused by factors that do not change gene expression levels, including non-synonymous variants or drug inhibition of the enzyme (i.e., sorivudine and brivudin). Therefore, other strategies have to be considered to detect reduced DPD activity among patients. For this reason, the measurement of endogenous uracil/5,6-dihydrouracil ratio $(\mathrm{U} / \mathrm{UH} 2)$ prior to the administration of 5-FU or capecitabine has been widely investigated as a surrogate marker of DPD activity. Indeed, enzyme deficiency impairs the metabolic breakdown of pyrimidines, thus causing an accumulation of uracil that can be detected in biological matrices (plasma or urine) by HPLC. Recent studies found that elevated levels of uracil in plasma were significantly associated with impaired clearance of 5-FU and development of 5-FU-related toxicity [34, 35]. Hence, the determination of the $\mathrm{U} / \mathrm{UH} 2$ ratio, measured before 5-FU administration, may be clinically useful to prevent the occurrence of adverse reactions. 


\section{$2-{ }^{13} \mathrm{C}$-uracil metabolism and breath test}

Another strategy is based on the evaluation of $2{ }^{13} \mathrm{C}$-uracil metabolism by measuring the exhaled levels of ${ }^{13} \mathrm{CO}_{2}$ and ${ }^{12} \mathrm{CO}_{2}$ using infrared spectrophotometry. Several studies demonstrated that reduced levels of exhaled ${ }^{13} \mathrm{CO}_{2}$ were strongly correlated to partial or complete DPD deficiency, as measured by the radiochemical enzyme assay [36]. The breath test is non-invasive and rapid, and gives accurate results of DPD activity within an hour after the intake of $2{ }^{13} \mathrm{C}$-uracil. Other advantages are represented by the stability of exhaled isotopes and the easy of access of the analysis method used to measure $2-{ }^{13} \mathrm{C}$-uracil, as it is the same used for the H. pylori breath test.

However, an important disadvantage of this method is the limited availability of the test substrate, and the interassay variability among different laboratories. The latter issue may be critical if this and other tests gain widespread use in the clinical practice. Finally, standardized threshold values to separate normal patients from those with DPD deficiency are still lacking.

5-FU and 5-FDHU pharmacokinetics after 5-FU standard or reduced test dose

Other groups of researchers attempted to investigate DPD deficiency trough the measurement of plasma levels of 5FU and/or its catabolites. Several studies have demonstrated that alterations in 5-FU and 5-FDHU pharmacokinetics were significantly associated with treatment tolerability. In colorectal cancer patients receiving 5-FU-based adjuvant chemotherapy, clearance (CL) and area under the time/ concentration curve (AUC) of the drug were significantly different between patients who reported moderate-tosevere toxicities and those who tolerated well the treatment with fluoropyrimidines [28, 36]. These differences suggested that severe adverse events were related to changes in drug systemic exposure and elimination. As a matter of fact, the ratio between the AUC values of the substrate 5FU and the metabolic product 5-FDHU was related to the severity of toxicity better than any other pharmacokinetic parameter, and the AUC ratio was predictive for the risk of gastrointestinal toxicity. Furthermore, results suggested that the pharmacokinetic analysis was more reliable and less prone to variability than the enzyme activity assay. The major drawback of pharmacokinetic-based strategies depends on the evaluation of patient metabolic status upon the first administration of therapeutic doses of 5-FU, hence exposing subjects to possible severe toxicities. In order to improve the safety of pharmacokinetic-based identification of patients at risk for severe or life-threatening toxicities, a reduced dose test was elaborated and subsequently validated in colorectal cancer patients [37-39]. This approach helps identify patients at major risk of 5-FU-associated toxicities on the basis of systemic pharmacokinetic markers (i.e., AUC, CL, elimination half-life) of both 5-FU and its inactive metabolite $5-\mathrm{FDHU}$ by using a reduced drug dose that has been demonstrated to be safe for the patient. Finally, a recent report, using a small number of patients, suggested the measurement of plasma levels of FBAL, the end product of 5-FU in the catabolic pathway, by HPLC to assess DPD enzyme activity [40]. Decreased levels of FBAL were significantly correlated with reduced DPD activity, as measured by radio-assay. However, this assay requires the administration of at least a test dose of a fluoropyrimidine.

\section{DPD genotyping}

The search for genetic variants of DPD and their association with enzyme activity represents a clinically useful approach to investigate DPD deficiency. The advantage over other methods consists in the requirement of a small blood sample for DNA extraction that can be stored at room temperature and it does not require the precautions necessary for an enzymatic test. The detection of DPD sequence variants is mainly carried out by denaturing HPLC analysis and then by automatic sequencing [41]. The gene encoding DPD is located in human chromosomal region $1 \mathrm{p} 22$ and is formed by 23 exons of approximately $950 \mathrm{~kb}[42,43]$. Over 30 single nucleotide polymorphisms (SNPs) and deletion/insertion mutations have been identified within DPD (Fig. 4, Tables 1 and 2), although most of these variants have no functional consequences on enzymatic activity. The most important variant associated with substantially reduced enzyme activity in heterozygous patients and lack of detectable activity in homozygous subjects is the IVS14+1G >A (DPYD*2A), which has been found in up to $40-50 \%$ of subjects with partial or complete DPD deficiency [20, 44, 45] (Table 2). This mutation changes the invariant splice donor site from GT to AT, leading to the skipping of exon 14 immediately upstream of the mutated splice donor site in the process of DPD premRNA splicing. As a result, the mature DPD mRNA lacks a 165-bp segment encoding the amino acids 581-635 and the mutant DPD protein has no residual enzymatic activity, as no significant DPD-dependent metabolism is measured in patients with this polymorphism. A toxic death after the administration of capecitabine was described by Largillier et al. in a breast cancer patient who displayed a deficient phenotype and was demonstrated to be heterozygous for the IVS14+1G $>$ A polymorphism [46]. Many other polymorphisms have been reported as responsible of the severe toxicity after 5-FU administration (Table 1). van Kuilenburg et al. analyzed the DPD gene of 14 patients with a reduced DPD activity and the analysis revealed the presence of 
Fig. 4 Schematic representation of DPD gene structure and polymorphisms

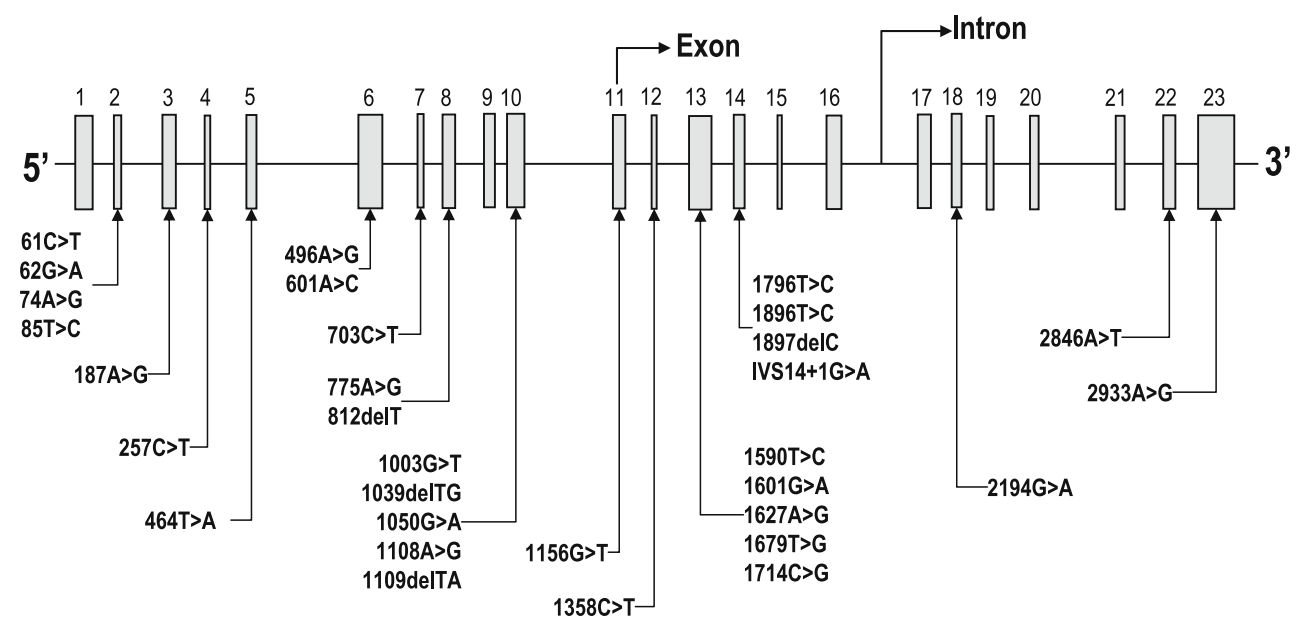

mutations in 11 out of 14 patients and five patients showed multiple mutations in the coding region of the DPD gene. In four patients it was detected the missense mutation $85 \mathrm{~T}>\mathrm{C}$, whereas the splice site mutation IVS14+1G $>$ A was detected in six subjects. One patient was homozygous for the 2194G> A mutation, whereas three patients were heterozygous for the $1627 \mathrm{~A}>\mathrm{G}$ mutation and two patients were homozygous for the $496 \mathrm{~A}>\mathrm{G}$ and $2846 \mathrm{~A}>\mathrm{T}$. The $2846 \mathrm{~A}>\mathrm{T}$ variant has also been detected in a patient with a complete DPD deficiency [47, 48]. Gross et al. analyzed four individuals with symptoms of 5-FU-related toxicity and detected six sequence variants in the DPD. Among them, $775 \mathrm{~A}>\mathrm{G}$ was found in a breast cancer patient who had received CMF polychemotherapy and displayed severe toxicity. None of a control cohort of 157 healthy individuals displayed this variant, suggesting that the base pair change might be a deleterious mutation and the novel mutation was present together with the known $85 \mathrm{~T}>\mathrm{C}$. Another patient, treated with $\mathrm{CMF}$ due to invasive breast carcinoma, carried four missense muta- tions $85 \mathrm{~T}>\mathrm{C}, 496 \mathrm{~A}>\mathrm{G}, 1601 \mathrm{G}>\mathrm{A}$, and $1627 \mathrm{~A}>\mathrm{G}$. The compound heterozygote genotype did not occur in any of the 157 control samples and had not been described previously. The combination of $496 \mathrm{~A}>\mathrm{G}$ and $1601 \mathrm{G}>\mathrm{A}$ was demonstrated in a colon cancer patient showing unexpected toxicity upon 5-FU administration. In the third patient, with invasive breast carcinoma given 5-FU, epirubicin and cyclophosphamide, $85 \mathrm{~T}>\mathrm{C}$ and the silent mutation $1896 \mathrm{~T}>\mathrm{C}$ were found in the coding area of DPD [41]. A meta-analysis of over 1200 patients suggested that more than $30 \%$ of patients treated with 5-FU experienced substantial drug-related toxicity [49]. However, genetic variants of DPD are not always associated with severe 5-FU toxicity. Table 1 and 2 report some of the more common SNPs identified in the DPD. While some mutations are directly responsible for severe 5-FU side effects, for example the IVS14+1G $>$ A which is always associated with high toxicity in cancer patients, others, like the $1601 \mathrm{G}>\mathrm{A}$ polymorphism, result in 5-FU related toxicity only in combination with other genetic variants such as the
Table 1 DPD polymorphisms of unknown significance

\begin{tabular}{|c|c|c|}
\hline Polymorphism & Localization & Reference \\
\hline $61 \mathrm{C}>\mathrm{T}, 62 \mathrm{G}>\mathrm{A}, 74 \mathrm{~A}>\mathrm{G}$ & exon 2 & {$[47,53]$} \\
\hline $187 \mathrm{~A}>\mathrm{G}$ & exon 3 & {$[55]$} \\
\hline $257 \mathrm{C}>\mathrm{T}$ & exon 4 & {$[47]$} \\
\hline $601 \mathrm{~A}>\mathrm{C}$ & exon 6 & [47] \\
\hline IVS6-29G $>\mathrm{T}$ & splice site variant intron 6 & {$[54]$} \\
\hline $703 \mathrm{C}>\mathrm{T}$ & exon 7 & {$[59]$} \\
\hline $775 \mathrm{~A}>\mathrm{G}, 812 \mathrm{delT}$ & exon 8 & {$[41,53]$} \\
\hline $1003 \mathrm{G}>\mathrm{T}, 1039-1042$ delTG, $1050 \mathrm{G}>\mathrm{A}, 1108 \mathrm{~A}>\mathrm{G}$ & exon 10 & {$[47,53,55]$} \\
\hline IVS10-15 $\mathrm{T}>\mathrm{C}$ & splice site variant intron 10 & {$[21]$} \\
\hline $1156 \mathrm{G}>\mathrm{T}$ & exon 11 & {$[53]$} \\
\hline $1358 \mathrm{C}>\mathrm{T}$ & exon 12 & {$[22]$} \\
\hline $1590 \mathrm{~T}>\mathrm{C}, 1679 \mathrm{~T}>\mathrm{G}, 1714 \mathrm{C}>\mathrm{G}$ & exon13 & {$[47,53,58]$} \\
\hline $1796 \mathrm{~T}>\mathrm{C}, 1897 \mathrm{delC}$ & exon 14 & {$[23,53]$} \\
\hline $2933 A>G$ & exon 23 & [47] \\
\hline
\end{tabular}


Table 2 DPD polymorphisms associated with toxicity by fluoropyrimidines

\begin{tabular}{lll}
\hline Polymorphism & Localization & Reference \\
\hline $85 \quad \mathrm{~T}>\mathrm{C}$ & exon 2 & {$[47]$} \\
$464 \quad \mathrm{~T}>\mathrm{A}$ & exon 5 & {$[24]$} \\
$496 \mathrm{~A}>\mathrm{G}$ & exon 6 & {$[21]$} \\
$1109 \mathrm{delTA}$ & exon 10 & {$[21]$} \\
$1601 \mathrm{G}>\mathrm{A}$, 1627A $>\mathrm{G}$ & exon 13 & {$[41,57]$} \\
$1896 \quad \mathrm{~T}>\mathrm{C}$ & exon 14 & {$[56]$} \\
IVS14+1G $>$ A & splice site variant intron 14 & {$[56]$} \\
$2194 \mathrm{G}>\mathrm{A}$ & exon 18 & {$[47]$} \\
$2846 \mathrm{~A}>\mathrm{T}$ & exon 22 & {$[21]$} \\
\hline
\end{tabular}

$496 \mathrm{~A}>\mathrm{G}$ [41]. Recent studies have also suggested that epigenetic factors may influence DPD activity, such as an aberrant methylation of the DPD promoter gene, which was found to cause a partially DPD deficient phenotype [50-52].

\section{Application of DPD screening in the clinical setting: is it feasible?}

While the uracil breath test and plasma uracil quantitation to stratify patients for their risk of incurring in fluoropyrimidine-induced toxicity are useful with some im- portant limitations, the genomic approach appears to be superior for indirect assessment of DPD. Although most of the informations come from case reports or a few published articles, the available clinical evidences obtained either prospectively or retrospectively clearly identify IVS14+1G> $\mathrm{A}$ as the most important mutation causing severe toxicity or even death. Therefore, IVS14 $+1 \mathrm{G}>\mathrm{A}$ testing should be performed in patients before the first administration of fluoropyrimidines or in those who suffered from severe toxicities. The preliminary screening of other variants associated with less-severe adverse drug reactions before the commencement of therapy is not justified. The following approach may be suggested for patients never treated with fluoropyrimidines or with fluoropyrimidine-induced toxicity (Fig. 5). New patients are screened for the most important and potentially lethal IVS14+1G>A mutation. If they are negative, they may be treated with standard doses of the drugs and then screened afterwards for other DPD variants if they display toxicity after the first doses of treatment. If they are heterozygous for IVS14 $+1 \mathrm{G}>\mathrm{A}$, the dose should be reduced at least empirically or, preferably, after accurate assessment of residual metabolic activity by DPD enzyme activity of 5-FU-test dose (Fig. 5). If a patient treated with fluoropyrimidines displays toxicity, he should be screened at least for the variants listed in Table 1. If heterozygous for IVS14 $+1 \mathrm{G}>\mathrm{A}$ or hetero- or homozygous for other variants, he should be treated by empirically reduced drug dose of after phenotypic assessment (enzymatic DPD activity measure-
Fig. 5 Proposed algorithm for screening DPD deficiency and dose adjustment in patients

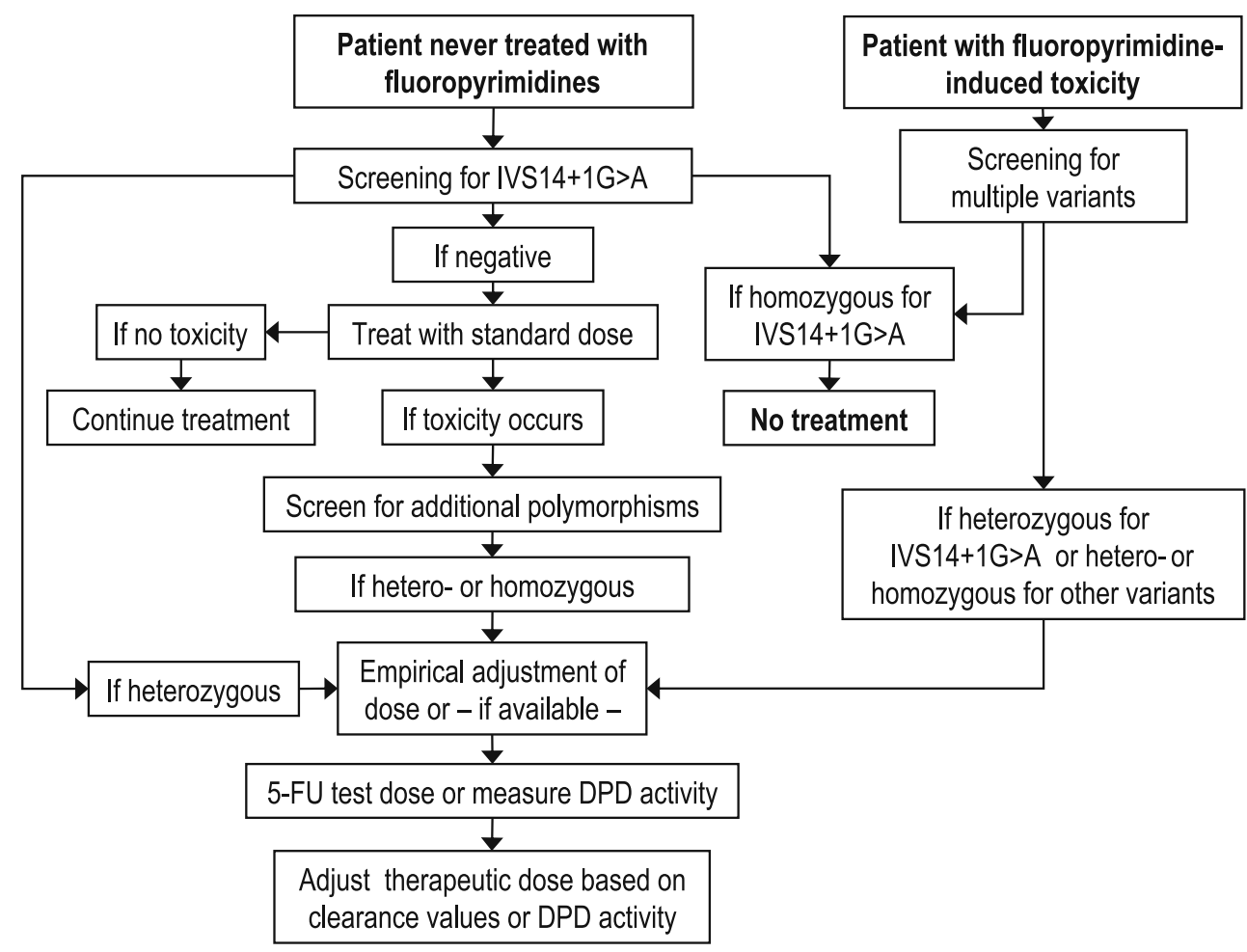


ment or 5-FU pharmacokinetics). In all cases, patients homozygous for IVS14+1G $>$ A must not be treated with fluoropyrimidines.

\section{Conclusion and outlook}

DPD deficiency plays an important role in the development of fluoropyrimidine-associated severe toxicity. However, no consensus exists on the clinical application of DPD screening in the population of patients candidates to fluoropyrimidine therapy. Herewith we propose the use of a pharmacogenetic approach to identify patients at risk of developing major toxicities and we present an algorithm based on the preliminary screening of the most dangerous polymorphism associated with severe toxicity and potentially death by 5 -FU and derivatives. The screening of additional variants may be performed if toxicity occurs after the administration of fluoropyrimidines to patients negative for IVS14+1G $>$ A.

Owing to the widespread use of these anticancer agents in the treatment of patients and the availability of reliable methods, the screening for the DPD variants should be considered before the start of treatment to prevent the occurrence of potentially lethal adverse reactions, particularly in the adjuvant setting and in borderline patients. Due to the multiple genetic variants of DPD gene, highthroughput analysis platforms (i.e., SNP genotyping by TaqMan, proteomic analysis) should be available in clinical laboratories to make the access to DPD analysis easier for the clinician with the goal of preventing toxic deaths depending on inherited deficiency of DPD.

\section{References}

1. Das P, Lin EH, Bhatia S, et al. Preoperative chemoradiotherapy with capecitabine versus protracted infusion 5-fluorouracil for rectal cancer: a matched-pair analysis. Int $\mathrm{J}$ Radiat Oncol Biol Phys. 2006;66(5):1378-83.

2. Schmoll H-J, Cartwright T, Tabernero J, et al. Phase III trial of capecitabine plus oxaliplatin as adjuvant therapy for stage III colon cancer: a planned safety analysis in 1864 patients. J Clin Oncol. 2007;25(1):102-9.

3. Walko CM, Lindley C. Capecitabine: a review. Clin Ther. 2005;27 (1):23-44.

4. van Kuilenburg ABP. Dihydropyrimidine dehydrogenase and the efficacy and toxicity of 5-fluorouracil. Eur J Cancer. 2004;40 (7):939-50.

5. de Bono JS, Twelves CJ. The oral fluorinated pyrimidines. Invest New Drugs. 2001;19(1):41-59.

6. Ishikawa T, Utoh M, Sawada N, et al. Tumor selective delivery of 5-fluorouracil by capecitabine, a new oral fluoropyrimidine carbamate, in human cancer xenografts. Biochem Pharmacol. 1998;55(7):1091-7.
7. Mattison LK, Soong R, Diasio RB. Implications of dihydropyrimidine dehydrogenase on 5-fluorouracil pharmacogenetics and pharmacogenomics. Pharmacogenomics. 2002;3(4):485-92.

8. Gardiner SJ, Begg EJ, Robinson BA. The effect of dihydropyrimidine dehydrogenase deficiency on outcomes with fluorouracil. Adverse Drug React Toxicol Rev. 2002;21(1-2):1-16.

9. van Kuilenburg ABP, De Abreu RA, van Gennip AH. Pharmacogenetic and clinical aspects of dihydropyrimidine dehydrogenase deficiency. Ann Clin Biochem. 2003;40(Pt 1):41-5.

10. Etienne MC, Lagrange JL, Dassonville O, et al. Population study of dihydropyrimidine dehydrogenase in cancer patients. J Clin Oncol. 1994;12(11):2248-53.

11. Lu Z, Zhang R, Carpenter JT, Diasio RB. Decreased dihydropyrimidine dehydrogenase activity in a population of patients with breast cancer: implication for 5-fluorouracil-based chemotherapy. Clin Cancer Res. 1998;4(2):325-9.

12. Sohn D-R, Cho MS, Chung P-J. Dihydropyrimidine dehydrogenase activity in a Korean population. Ther Drug Monit. 1999;21(2):152-4.

13. Yamaguchi K, Arai Y, Kanda Y, Akagi K. Germline mutation of dihydropyrimidine dehydrogenase gene among a Japanese population in relation to toxicity to 5-fluorouracil. Jpn J Cancer Res. 2001;92(3):337-42.

14. Ogura K, Ohnuma T, Minamide Y, et al. Dihydropyrimidine dehydrogenase activity in 150 healthy Japanese volunteers and identification of novel mutations. Clin Cancer Res. 2005;11 (14):5104-11.

15. Saif MW, Mattison L, Carollo T, et al. Dihydropyrimidine dehydrogenase deficiency in an Indian population. Cancer Chemother Pharmacol. 2006;58(3):396-401.

16. Hamdy SI, Hiratsuka M, Narahara K, et al. Allele and genotype frequencies of polymorphic cytochromes P450 (CYP2C9, CYP2C19, CYP2E1) and dihydropyrimidine dehydrogenase (DPYD) in the Egyptian population. Br J Clin Pharmacol. 2002;53(6):596-603.

17. Salgueiro N, Veiga I, Fragoso M, et al. Mutations in exon 14 of dihydropyrimidine dehydrogenase and 5-fluoruracil toxicity in Portuguese colorectal cancer patients. Genet Med. 2004;6(2):102-7.

18. Seck K, Riemer S, Kates R, et al. Analysis of the DPYD gene implicated in 5-fluorouracil catabolism in a cohort of caucasian individuals. Clin Cancer Res. 2005;11(16):5886-92.

19. Mattison LK, Fourie J, Desmond RA, et al. Increased prevalence of dihydropyrimidine dehydrogenase deficiency in AfricanAmericans compared with caucasians. Clin Cancer Res. 2006;12 (18):5491-5.

20. Van Kuilenburg ABP, Meinsma R, Zoetekouw L, Van Gennip $\mathrm{AH}$. Increased risk of grade IV neutropenia after administration of 5-fluorouracil due to a dihydropyrimidine dehydrogenase deficiency: high prevalence of the IVS14 $+1 \mathrm{G}>\mathrm{A}$ mutation. Int $\mathrm{J}$ Cancer. 2002;101(3):253-8.

21. Gross E, Busse B, Riemenschneider M, et al. Strong association of a common dihydropyrimidine dehydrogenase gene polymorphism with fluoropyrimidine-related toxicity in cancer patients. PLoS ONE. 2008;3(12):e4003.

22. Shahrokni A, Rajebi MR, Harold L, Saif MW. Cardiotoxicity of 5-fluorouracil and capecitabine in a pancreatic cancer patient with a novel mutation in the dihydropyrimidine dehydrogenase gene. JOP. 2009;10(2):215-20.

23. Ofverholm A, Arkblad E, Skrtic S, et al. Two cases of 5fluorouracil toxicity linked with gene variants in the DPYD gene. Clin Biochem. 2010;43(3):331-4.

24. Morel A, Boisdron-Celle M, Fey L, et al. Identification of a novel mutation in the dihydropyrimidine dehydrogenase gene in a patient with a lethal outcome following 5-fluorouracil administration and the determination of its frequency in a population of 500 patients with colorectal carcinoma. Clin Biochem. 2007;40(12):11-7. 
25. Lu Z, Zhang R, Diasio RB. Dihydropyrimidine dehydrogenase activity in human peripheral blood mononuclear cells and liver Population characteristics, newly identified deficient patients, and clinical implication in 5-fluorouracil chemotherapy. Cancer Res. 1993;53(22):543-8

26. Chazal M, Etienne MC, Renee N, et al. Link between dihydropyrimidine dehydrogenase activity in peripheral blood mononuclear cells and liver. Clin Cancer Res. 1996;2(3):507-10.

27. van Kuilenburg ABP, Van Lenthe H, Van Gennip AH. Radiochemical assay for determination of dihydropyrimidinase activity using reversed-phase high performance liquid chromatography. J Chromatogr B. 1999;729(1-2):307-14.

28. Di Paolo A, Danesi R, Falcone A, et al. Relationship between5-fluorouracil disposition, toxicity and dihydropyrimidine dehydrogenase activity in cancer patients. Ann Oncol. 2001;12 (9):1301-6.

29. Van Kuilenburg AB, van Lenthe H, Blom MJ, et al. Profound variation in dihydropyrimidine dehydrogenase activity in human blood cells: major implications for the detection of partly deficient patients. Br J Cancer. 1999;79(3-4):620-6.

30. Johnson MR, Wang K, Smith JB, et al. Quantitation of dihydropyrimidine dehydrogenase expression by real-time reverse transcription polymerase chain reaction. Anal Biochem. 2000;278(2):175-84.

31. Seck K, Riemer S, Kates R, et al. Analysis of the DPYD gene implicated in 5-fluorouracil catabolism in a cohort of caucasian individuals. Clin Cancer Res. 2005;11(16):5886-92.

32. Johnson MR, Wang K, Diasio RB. Profound dihydropyrimidine dehydrogenase deficiency resulting from a novel compound heterozygote genotype. Clin Cancer Res. 2002;8(3):768-74.

33. Boisdron-Celle M, Remaud G, Traore S, et al. 5-Fluorouracilrelated severe toxicity: a comparison of different methods for the pretherapeutic detection of dihydropyrimidine dehydrogenase deficiency. Cancer Lett. 2007;249(2):271-82.

34. Jiang H, Lu J, Jiang J, Hu P. Important role of the dihydrouracil/ uracil ratio in marked interpatient variations of fluoropyrimidine pharmacokinetics and pharmacodynamics. J Clin Pharmacol. 2004;44(11):1260-72.

35. Garg M, Sevester JC, Sakoff JA, Ackland SP. Simple liquid chromatographic method for the determination of uracil and dihydrouracil plasma levels: a potential pretreatment predictor of 5-fluorouracil toxicity. J Chromatogr B. 2002;774(2):223-30.

36. Mattison LK, Ezzeldin H, Carpenter M, et al. Rapid identification of dihydropyrimidine dehydrogenase deficiency by using a novel 2-13C-uracil breath test. Clin Cancer Res. 2004;10(8):2652-8.

37. Di Paolo A, Ibrahim T, Danesi R, et al. Relationship between plasma concentrations of 5- fluorouracil and 5-fluoro-5, 6dihydrouracil and toxicity of 5-fluorouracil infusions in cancer patients. Ther Drug Monit. 2002;24(5):588-93.

38. Bocci G, Danesi R, Di Paolo A, et al. Comparative pharmacokinetic analysis of 5-fluorouracil and its major metabolite 5-fluoro5, 6-dihydrouracil after conventional and reduced test dose in cancer patients. Clin Cancer Res. 2000;6(8):3032-7.

39. Bocci G, Barbara C, Vannozzi F, et al. A pharmacokinetic-based test to prevent severe 5- fluorouracil toxicity. Clin Pharmacol Ther. 2006;80(4):384-95.

40. Furuhata T, Kawakami M, Okita K, et al. Plasma level of a 5 fluorouracil metabolite, fluorobeta-alanine correlates with dihydropyrimidine dehydrogenase activity of peripheral blood mononuclear cells in 5-fluorouracil treated patients. J Exp Clin Cancer Res. 2006;25(1):79-82.

41. Gross E, Ullrich T, Seck K, et al. Detailed analysis of five mutations in dihydropyrimidine dehydrogenase detected in cancer patients with 5-fluorouracil-related side effects. Hum Mutat. 2003;22(6):498
42. Takai S, Fernandez-Salguero P, Kimura S, et al. Assignment of the human dihydropyrimidine dehydrogenase gene (DPYD) to chromosome region $1 \mathrm{p} 22$ by fluorescence in situ hybridization. Genomics. 1994;24(3):613-4.

43. Wei X, Elizondo G, Sapone A, et al. Characterization of the human dihydropyrimidine dehyrdogenase gene. Genomics. 1998;51(3):391-400.

44. van Kuilenburg ABP, Vreken P, Abeling NGGM, et al. Genotype and phenotype in patients with dihydropyrimidine dehydrogenase deficiency. Hum Genet. 1999;104(1):1-9.

45. Wei X, McLeod HL, McMurrough J, et al. Molecular basis of the human dihydropyrimidine dehydrogenase deficiency and 5 fluorouracil toxicity. J Clin Invest. 1996;98(3):610-5.

46. Largillier R, Etienne-Grimaldi MC, Formento JL, et al. Pharmacogenetics of capecitabine in advanced breast cancer patients. Clin Cancer Res. 2006;12(18):5496-502.

47. van Kuilenburg AB, Haasjes J, Richel DJ, et al. Clinical implications of dihydropyrimidine dehydrogenase (DPD) deficiency in patients with severe 5-fluorouracil-associated toxicity: identification of new mutations in the DPD gene. Clin Cancer Res. 2000;6(12):4705-12.

48. Gamelin EC, Boisdron-Celle M. Dose monitoring of 5- fluorouracil in patients with colorectal or head and neck cancer: state of the art. Crit Rev Oncol Hematol. 1999;30(1):71-9.

49. Meta-Analysis Group In Cancer. Toxicity of fluorouracil in patients with advanced colorectal cancer: effect of administration schedule and prognostic factors. J Clin Oncol. 1998;16(11):3537-41.

50. Noguchi T, Tanimoto K, Shimokuni T, et al. Aberrant methylation of DPYD promoter, DPYD expression, and cellular sensitivity to 5fluorouracil in cancer cells. Clin Cancer Res. 2004;10(20):7100-7.

51. Ezzeldin HH, Lee AM, Mattison LK, Diasio RB. Methylation of the DPYD promoter: an alternative mechanism for dihydropyrimidine dehydrogenase deficiency in cancer patients. Clin Cancer Res. 2005;11(24 Pt 1):8699-705.

52. Yu J, McLeod HL. Methylation of the DPYD promoter and dihydropyrimidine dehydrogenase deficiency. Clin Cancer Res. 2006;12(12):3864.

53. He YF, Wei W, Zhang X, et al. Analysis of the DPYD gene implicated in 5-fluorouracil catabolism in Chinese cancer patients. J Clin Pharm Ther. 2008;33(3):307-14.

54. Ben Fredj R, Gross E, Chouchen L, et al. Mutational spectrum of dihydropyrimidine dehydrogenase gene (DPYD) in the Tunisian population. C R Biol. 2007;330(10):764-9.

55. Kleibl Z, Fidlerova J, Kleiblova P, et al. Influence of dihydropyrimidine dehydrogenase gene (DPYD) coding sequence variants on the development of fluoropyrimidine-related toxicity in patients with high-grade toxicity and patients with excellent tolerance of fluoropyrimidine-based chemotherapy. Neoplasma. 2009;56 (4):303-16.

56. Mercier C, Ciccolini J. Severe or lethal toxicities upon capecitabine intake: is DPYD genetic polymorphism the ideal culprit? Trends Pharmacol Sci. 2007;28(12):597-8.

57. Zhang $\mathrm{H}$, Li YM, Zhang $\mathrm{H}$, Jin $\mathrm{X}$. DPYD*5 gene mutation contributes to the reduced DPYD enzyme activity and chemotherapeutic toxicity of 5-FU: results from genotyping study on 75 gastric carcinoma and colon carcinoma patients. Med Oncol. 2007;24(2):251-8.

58. Morel A, Boisdron-Celle M, Fey L, et al. Clinical relevance of different dihydropyrimidine dehydrogenase gene single nucleotide polymorphisms on 5-fluorouracil tolerance. Mol Cancer Ther. 2006;5(11):2895-904.

59. Vreken P, Van Kuilenburg AB, Meinsma R, van Gennip AH Dihydropyrimidine dehydrogenase (DPD) deficiency: identification and expression of missense mutations $\mathrm{C} 29 \mathrm{R}, \mathrm{R} 886 \mathrm{H}$ and R235W. Hum Genet. 1997;101(3):333-8. 